\title{
Training cognitive control in older adults with the Space Fortress game: the role of training instructions and basic motor ability
}

\author{
Helena M. Blumen ${ }^{1}$, Daniel Gopher ${ }^{2}$, Joshua R. Steinerman ${ }^{3}$ and Yaakov Stern ${ }^{1 *}$ \\ Cognitive Neuroscience Division, Taub Institute, Columbia University, New York, NY, USA \\ 2 Industrial Engineering and Management, Technion - Israel Institute of Technology, Haifa, Israel \\ ${ }^{3}$ Department of Neurology, Albert Einstein College of Medicine, New York, NY, USA
}

Edited by:

Arthur F. Kramer, University of Illinois at Urbana-Champaign, USA

\section{Reviewed by:}

David Strayer, University of Utah, USA

Louis Bherer, Institut Universitaire de

Gériatrie de Montréal, Canada

Walter Richard Boot, Florida State

University, USA

*Correspondence:

Yaakov Stern, Columbia University, P\&S Box 16, 630 W. 168th Street,

New York, NY 10032, USA.

e-mail:ys11@columbia.edu
This study examined if and how cognitively healthy older adults can learn to play a complex computer-based action game called the Space Fortress (SF) as a function of training instructions [Standard vs. Emphasis Change (EC); e.g., Gopher et al., 1989] and basic motor ability. A total of 35 cognitively healthy older adults completed a 3-month SF training program with three SF sessions weekly. Twelve 3-min games were played during each session. Basic motor ability was assessed with an aiming task, which required rapidly rotating a spaceship to shoot targets. Older adults showed improved performance on the SF task over time, but did not perform at the same level as younger adults. Unlike studies of younger adults, overall SF performance in older adults was greater following standard instructions than following EC instructions. However, this advantage was primarily due to collecting more bonus points and not - the primary goal of the game - shooting and destroying the fortress, which in contrast benefited from EC instructions. Basic motor ability was low and influenced many different aspects of SF game learning, often interacted with learning rate, and influenced overall SF performance. These findings show that older adults can be trained to deal with the complexity of the SF task but that overall SF performance, and the ability to capitalize on EC instructions, differs when a basic ability such as motor control is low. Hence, the development of this training program as a cognitive intervention that can potentially compensate for age-related cognitive decline should consider that basic motor ability can interact with the efficiency of training instructions that promote the use of cognitive control (e.g., EC instructions) - and the confluence between such basic abilities and higher-level cognitive control abilities should be further examined.

Keywords: cognitive training, Space Fortress game, emphasis change, motor control, cognitive interventions, cognitive aging

\section{INTRODUCTION}

The current study examined if and how cognitively healthy older adults can learn to play a complex computer-based action game called the Space Fortress (SF). Playing this game demands a considerable amount of cognitive control. Cognitive or executive control refers to a set of processes that monitor and coordinate complex behaviors such as planning, abstract thinking, reasoning, and the selection and inhibition of appropriate actions (Norman and Shallice, 1986). Cognitive control processes are presumably utilized in a number of different laboratory tasks and real-world activities and are particularly affected by aging (e.g., Moscovitch and Winocur, 1992). Training cognitive control may also be critical for demonstrating transfer of learning to other laboratory tasks and real-world activities, and are therefore of particular interest in the development of new cognitive interventions. The main goal of this study was to examine the role of training instructions during SF game learning in cognitively healthy older adults. To this end, standard game play instructions were compared to training instructions that encouraged individuals to explore alternative learning strategies - i.e., Emphasis Change (EC) instructions (Gopher et al.,
1989). The second goal of this study was to examine the role of basic motor control abilities in how cognitively healthy older adults learn to play the SF game. To this end, basic motor ability was assessed prior to SF game training with a short psychomotor task - i.e., the aiming task (e.g., Gopher et al., 1989; Mané and Donchin, 1989).

\section{COGNITIVE CONTROL AND COGNITIVE INTERVENTIONS}

The development of new cognitive interventions that can be effectively applied to compensate for age-related cognitive decline is of key concern to contemporary aging research (see Green and Bavelier, 2008; Hertzog et al., 2008; Papp et al., 2009 for reviews). Early cognitive interventions demonstrated improved performance on the previously trained task but many failed to demonstrate transfer of learning to other laboratory tasks or real-world activities. For example, a meta-analysis of 33 studies of cognitively healthy older adults trained in the use of some mnemonic technique (such as the method of loci or imagery) concluded that performance improvements were largely specific to the previously trained mnemonic technique (Verhaeghen et al., 1992). Later cognitive interventions trained cognitive control and more 
successfully demonstrated transfer of learning to other laboratory tasks (Kramer et al., 1995; Bherer et al., 2005, 2008) and realworld activities (Willis et al., 2006). For example, training younger and older adults to control attention in one dual-task situation improved their ability to control attention in another dual-task situation (Kramer et al., 1995).

\section{COGNITIVE CONTROL AND COMPUTER-BASED GAMES}

Training cognitive control within the context of complex computer-based games may be particularly important for demonstrating transfer of learning to a number of different tasks. For example, a recent cognitive intervention that involved training older adults to play an off the shelf computer-based strategy game called Rise of Nations demonstrated improved performance on a number of different tasks including task-switching, working memory, visual short-term memory, and reasoning (Basak et al., 2008; for a similar finding in older adults see Drew and Waters, 1986; for similar findings on computer-based games in younger adults see Frederiksen and White, 1989; Gopher et al., 1989, 1994; Green and Bavelier, 2003, 2006, 2007). Cognitive interventions that utilize less complex computer-based games such a Super Tetris have also demonstrated transfer of learning but performance improvements seem to be limited to response times (Goldstein et al., 1997; Salthouse and Somberg, 1982; Clark et al., 1987). Thus, the complexity and dynamics of the computer-based game - and its overall demands for cognitive control - may be critical for demonstrating transfer of learning to a number of different tasks and real-world activities. Complex computer-based games also lend themselves to training programs that extend over weeks and or months, another factor that may be important for the development of more enduring and stable capabilities that can transfer to performance on realworld activities.

\section{COGNITIVE CONTROL AND THE SPACE FORTRESS GAME}

In the current study, we trained cognitively healthy older adults to play a complex computer-based action game, called the SF game. The SF game was originally developed to study complex skill acquisition (Gopher et al., 1989; Mané and Donchin, 1989) and was recently revised to accommodate contemporary computer environments (Shebilske et al., 2005, based on a version developed by Gopher et al., 1994 to train flight trainees). The primary goal of the SF game is to shoot missiles and destroy a space fortress while protecting your spaceship against damage. The spaceship and the missiles are controlled with a joystick. In addition to defending your spaceship against missiles shot by the space fortress the player has to defend herself against mines that intermittently appear on the screen. The player is also asked to monitor symbols that randomly appear on the screen and add to the participants' total score. Thus, the SF game is a fast-paced and dynamic computer game that requires divided attention, continuous and discrete motor control, visual search, working memory, long-term memory, resource management as well as decision-making processes. An individual's ability to control or switch their attention to these different task requirements is considered vital for successful SF game performance (Gopher et al., 1994; Arthur Jr. et al., 1995; Gopher, 2006). In other words, the SF game demands a considerable amount of cognitive control.
To date, older adults have not been trained to play the SF game. Thus, it remains unclear if and how older adults would learn to play this highly complex computer-based action game. The current study examined if and how cognitively healthy older adults learn to play the SF game. More specifically, we wanted to identify and determine the efficiency of the strategies that older adults adopt during training with the SF game - as a function of training instructions. Such an examination is critical for the further development of this training program as a cognitive intervention that can potentially compensate for age-related cognitive decline. In younger adults, training with the SF game has been shown to improve performance on other laboratory tasks (Fabiani et al., 1989; Frederiksen and White, 1989) as well as real-world tasks such a flight performance (Gopher et al., 1994). These findings from younger adults suggest that training cognitive control with the SF game can potentially be developed into a cognitive intervention that can compensate for age-related cognitive decline.

\section{THE SPACE FORTRESS GAME AND EMPHASIS CHANGE INSTRUCTIONS}

In younger adults, EC instructions - play the whole game but change your emphasis to different sub-components of the game at different points during training - are particularly beneficial to SF game performance. For example, training with EC instructions leads to better SF game performance than training with standard game instructions (i.e., play the whole game and develop learning strategies ad lib) in younger adults (Gopher et al., 1989). EC instructions are argued to encourage individuals to explore alternative learning strategies and further increase the demand for cognitive control. More specifically, EC instructions encourage individuals to concentrate on gaining control of the ship (ship handling emphasis), efficiently managing friend and foe mines (mine handling emphasis) or both. More specifically, controlling the ship in order to shoot and destroy the space fortress is expected to improve during ship handling emphasis while defending the ship against mines is expected to improve during mine handling emphasis.

One study suggest that hierarchical part-task instructions successively learning to play the SF game by practicing tasks that engage only one, or a few different SF task requirements - can lead to better SF performance than EC instructions (Fabiani et al., 1989). However, in this study SF performance of individuals trained with EC instructions were also more resistant to interference when a secondary task was introduced compared to the SF performance of individuals trained with hierarchical part-task instructions. This finding suggests that EC instructions increases the demand for cognitive control processes and may be particularly important for incorporating a secondary task and demonstrating transfer of learning (Gopher et al., 1989, 1994; Kramer et al., 1995; Gopher, 2007).

Additional evidence for the above proposition is obtained in a recent imaging study of younger adults trained to play the SF game with standard and EC instructions (Erickson et al., 2010). In this study, the volume of both the dorsal and the ventral striatum predicted initial SF acquisition but only the volume of the dorsal striatum predicted later improvements in SF performance. More importantly, the volume of the dorsal striatum predicted later improvements in SF performance following EC instructions but not following standard instructions. These findings are consistent 
with suggestion that the ventral striatum is involved in early reinforcement-related learning while the dorsal striatum is involved in both early and later motor-related and association-related learning and cognitive control (Desmond et al., 1998; Marie et al., 1999; Lawrence et al., 2000; Belin and Everitt, 2008). Thus, it seems reasonable to conclude that EC instructions during SF game learning place a particularly high demand on cognitive control. In the current study, we addressed this issue in cognitively healthy older adults by comparing SF game learning following EC instructions and standard game instructions. Although older adults have not been trained to play the SF game, they have been shown to benefit from EC instructions in a dual-task situation (Kramer et al., 1995).

\section{THE SPACE FORTRESS GAME AND BASIC MOTOR ABILITY}

The SF game involves manipulating a joy stick to steer, aim and shoot missiles with a space ship that moves in a frictionless environment. In other words, the dynamic control and aiming of the ship is an exceptionally difficult task that involves precise, discrete and continuous motor control. The aiming task is based upon the aiming component of the SF game and involves aiming and shooting missiles with a stationary space ship. In younger adults, the aiming task has been used as a threshold barrier to exclude participants who are less likely to successfully learn to play the SF game within a limited number of 10 training sessions. Typically, an aiming score less than 780 have been used as the criteria for exclusion (e.g., Gopher et al., 1989; Mané and Donchin, 1989). In the current study of cognitively healthy older adults we did not exclude participants with low aiming scores. This is because older adults were expected to have lower aiming scores than younger adults in general. In other words, excluding older adults with the same criteria as young adults would likely lead to the exclusion of a large amount of participants. Moreover, our overall aim was to investigate how older adults learn to play the SF game for the future development of a training program that can compensate for age-related cognitive decline. Thus, we used the aiming task to determine if it could be used as an indicator of who could benefit from such an intervention.

In sum, the current study examined if and how cognitively healthy older adults learn to play a complex computer-based action game called the SF, which demands a considerable amount of cognitive control. The goals of the current study were (1) to examine how cognitively healthy older adults learn to play the SF game as a function of training instructions - EC instructions vs. Standard game instructions and (2) to determine if and how basic levels of motor control abilities are related to how cognitively healthy older adults learn to play the SF game. We expected older adults to improve with SF gaming training but did not expect them to perform at the same level as younger adults. Given the young adult SF literature, we expected overall SF performance among older adults trained with EC instructions to be better than SF performance among older adults trained with standard instructions. Moreover, we expected sub-measures of SF performance related to ship handling and mine handling among older adults trained with EC instructions to be better than among older adults trained with standard instructionsbecause ship handling or mine handling is emphasized during EC instructions (see Table 2 and Statistical Approach for an explanation of these sub-measures).

\section{MATERIALS AND METHODS PARTICIPANTS}

A total of 40 cognitively healthy older adult participants were recruited via fliers, internet ads and visits to local senior centers. Participants that were trained to play the SF game were selected from a larger study that also involved a no SF game training condition. Participants had normal or corrected-to-normal vision and reported no past or current medical, neurological or psychiatric disorder. Participants were screened for dementia with the Mattis Dementia Rating Scale (DRS, Mattis, 1976). A total DRS score of 135 or higher were required for inclusion. Twenty participants were randomly assigned to each training condition - the EC condition and the Active Control (AC) condition. A total of five participants did not complete the 3-month training program - three from the EC condition and two from the AC condition, thus, 35 participants were included in the upcoming analyses. Additional demographic and screening information for each condition are provided in Table 1. Note that participants in the EC condition and the AC condition did not differ in terms of Age, Percent Female, Years of Education, or total DRS score $(t$ values $<1)$. All participants provided informed consent in accordance with the procedure of Columbia University Medical Center and were debriefed upon the completion of the experiment.

\section{APPARATUS}

The revised version of the SF game (Shebilske et al., 2005) was installed on three networked PC computers. The primary goal of this game was to shoot missiles and destroy a space fortress while protecting your spaceship against damage. The spaceship and the missiles were controlled by the player with a joystick (Flightstick Pro) and the spaceship moved in a frictionless environment. Any forward movement of the joystick made the spaceship accelerate and any lateral movement of the joystick made the spaceship rotate. If the ship was steered off the screen, it would reappear (or wrap) to the other side of the screen. If a button on top of the joystick was pressed, a missile was fired in the direction of the spaceship. In addition to defending the spaceship against missiles shot by the space fortress, the player had to defend themselves against mines that appeared on the screen every $4 \mathrm{~s}$. There were two types of mines: friend mines and foe mines. When friend mines were shot they were energized, ran into the space fortress and scored a hit. When foe mines appeared, the player had to switch the weapon system by pressing the right mouse button twice and then eliminate the mine with a missile. Prior to each

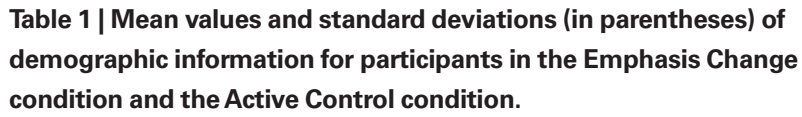
condition and the Active Control condition.

\begin{tabular}{lcr}
\hline Condition & Emphasis Change & Active Control \\
\hline Age & $66.35(4.55)$ & $65.56(4.05)$ \\
$\%$ Female & 0.59 & 0.50 \\
DRS (total) & $140.71(2.76)$ & $140.67(2.33)$ \\
Years of education & $15.65(2.55)$ & $16.28(2.61)$ \\
WAIS vocabulary (raw) & $55.47(7.74)$ & $54.38(12.46)$ \\
WTAR (raw) & $41.00(8.63)$ & $42.17(7.49)$
\end{tabular}


trial, the player was told what (3) letters signified foe mines. The space fortress had to be shot 10 times with more than $250 \mathrm{~ms}$ between each shot before it became vulnerable, then the space fortress could be destroyed with a double shot (i.e., two shots against the space fortress administered with no more than $250 \mathrm{~ms}$ between each shot). Bonus points could also be obtained by monitoring symbols that regularly popped up on the screen. Whenever the player saw $2 \$$ signs in a row, she could press the left mouse button and choose to receive bonus points or additional missiles. An approximate sketch of the SF game environment and its components are provided in Figure 1. One particularly nice feature of the SF game is that it can be programmed to compute sub-scores that reflect the different task requirements, in addition to overall or Total SF performance. These sub-scores can then be used to more closely analyze the different task requirements. A breakdown and explanation of the different sub-scores that were computed in our version of the SF game are provided in Table 2. Note that some of these sub-scores (Points, Control, Velocity, and Speed) also appear at the information panel at the bottom of the SF game environment, together with counters referring to the vulnerability of the space fortress, the letter associated with a mine, the interval between shots and the number of shots administered.

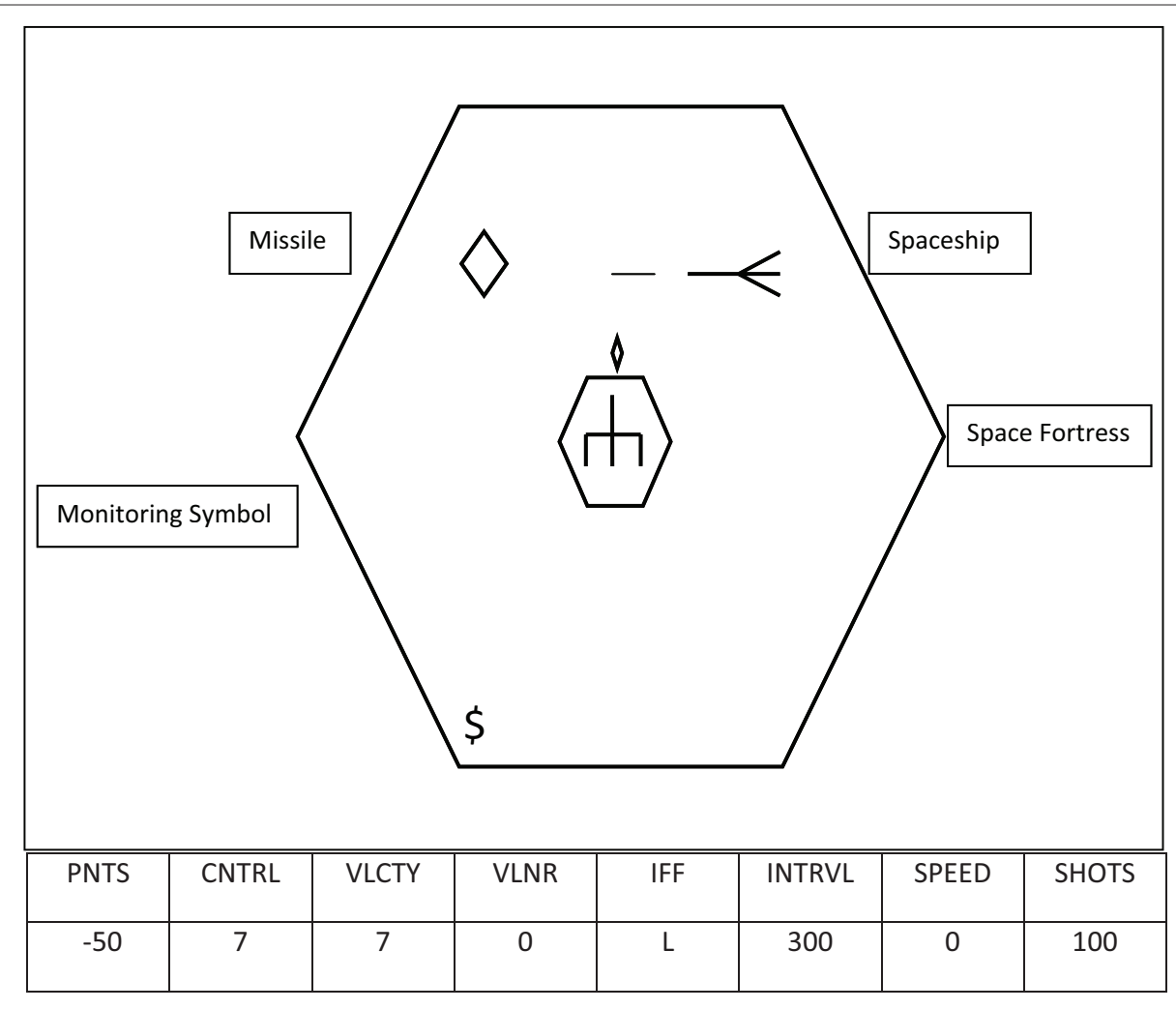

FIGURE 1|A Schematic of the revised Space Fortress game environment.

Table 2 | A description of each measure obtained from the space fortress game.

Total-Overall Space Fortress performance including Points, Velocity, Control, and Speed

\section{MAIN SUB-MEASURES}

Points - Increases from shooting and destroying the fortress and mines and decreases from damage and destruction of the player's ship

Velocity - Increases from flying the ship slowly and decreases from flying the ship fast

Control-Increases from keeping the ship within the hexagon and decreases from flying outside of the hexagon

Speed-Proportional to the efficiency of detecting and destroying mines

\section{POINT-GENERATING MEASURES}

Ship Damage by Fortress - Counts the number of times the ship is damaged by the space fortress ( -50 points)

Fortress Destruction - Counts the number of times the player is rewarded from destroying the fortress (+100 points)

Ship Damage by Mine - Counts the number of times the player's ship is damaged by missiles ( -50 points)

Fortress hit by Missile - Counts the number of times the space fortress is hit by a missile (+4 points)

Bonus Taken - Counts the number of times the player chooses bonus points as the reward following correct monitoring of two $\$$ sign in a row (+100 points) 


\section{PROCEDURE}

Prior to the first game training session, participants completed three trials of the aiming task that is based upon the aiming component of the SF game. During the aiming task, the player learns to aim and shoot missiles with a stationary spaceship. A total aiming score is obtained by adding the number of successful missiles shot within a given trial (points) and the speed of missiles shot with a given trial (speed).

\section{Space Fortress game training}

Our training program in general and our implementation of EC in particular, differed from previous SF game learning studies of younger adults. Typically, training in younger adults has consisted of 10, 1-h training sessions spaced over a couple of weeks (e.g., Gopher et al., 1989). We extended our training in older adults to three, 1-h sessions weekly for 12 weeks (i.e., a total of 36, 1-h sessions). Following standard game play instructions, young players assigned to the EC condition are instructed to focus on ship handling on some trials, mine handling on other trials or both ship handling and mine handling on some trials (Gopher et al., 1989). We also took additional measures to ensure that the older adults would stick with the 3 -month training program. During the first training session, each participant watched a flash tutorial about the SF game. The standard instructions were then read to them, allowing for questions along the way. Participants in the EC condition were also told to focus on flying the spaceship slowly and between the hexagons and maximize their Control and Velocity sub-score. During the first week of training, the experimenter also sat down with each participant (regardless of whether assigned to the EC or $\mathrm{AC}$ condition) and reviewed their performance after each game trial, not only in terms of their Total Score but also in terms of their sub-scores. Hence, both conditions were given EC instructions during the early training sessions because they were alerted to improve on different aspects of the game. However, on top of that, participants in the EC condition were told to focus on ship handling during some trials and on mine handling during other trials.

Participants completed a total of 36 SF training sessions over a 3-month period. Each training session consisted of 12,3-min game trials. Participants in the AC condition completed three training sessions, three times a week with standard game instructions (i.e., participants were told to maximize their Total game score, and use any strategy they felt would help them succeed). Participants in the EC condition also completed three training sessions three times a week for 12 weeks. However, during the first 10 trials in each session, participants were told to either focus on ship handling (i.e., participant were told to focus on flying the spaceship slowly and between the hexagons and maximize their Control and Velocity sub-score) or on mine handling (i.e., participants were told to focus on dealing with friend and foe mines and maximize their Speed sub-score). During the last two trials of each training session, participants in the EC condition also played the game with standard game instructions. Our implementation of EC instructions across training sessions can be summarized as follows. During the first 4 weeks of training, EC participants were asked to focus solely on ship handling. During week 4-6, EC participants were asked to focus on mine handling. Finally, during week 7-12 ship handling and mine handling were inter-mixed within sessions and trials. Note that during the 11th and the 12th trial in each session, both EC participants and AC participants received standard game instructions. The upcoming game play analyses were obtained from these trials.

\section{RESULTS AND DISCUSSION SUMMARY OF STATISTICAL ANALYSES}

We examined SF game performance as a function of the instructions given during training - EC instructions or (AC) standard game instructions - and basic motor control abilities in three different general linear models (GLMs). These models were composed of seven predictors: Week, Condition, Aiming, Week $\times$ Condition, Week $\times$ Aiming, Condition $\times$ Aiming, and Week $\times$ Condition $\times$ Aiming. In the first GLM, we analyzed Total SF performance in a repeated-measure analysis of covariance (ANCOVA) with Week (Week 1-12) as the repeated measure, Condition (EC vs. AC) as the between-subjects factor and Aiming as the covariate. In the second GLM, we used a repeated-measure multivariate ANCOVA (MANCOVA) to analyze the main submeasures of SF performance (Points, Velocity, Control, and Speed) with Week (Week 1-12) as the repeated measure, Condition (EC vs. AC) as the between-subjects factor and Aiming as the covariate. This second GLM allowed us to examine sub-scores of SF performance specifically related to ship handling (Velocity and Control) mine handling (Speed) as well as the total number of Points added from damaging the space fortress, destroying the space fortress and symbol monitoring and subtracted from damage to the spaceship created by mines and by the fortress. Finally, in the third GLM, we used a repeated-measure MANCOVA to analyze pointgenerating sub-measures of SF performance (Fortress Destroyed, Ship Damage by Fortress, Fortress Hit by Missile, Ship Damage by Mine, and Bonus Taken), with Week (Week 1-12) as the repeated measure, condition (EC vs. AC) as the between-subjects factor and Aiming as the covariate. This third GLM allowed us to examine points specifically generated from sub-measures related to ship handling (Fortress Destroyed, Ship Damage by Fortress, Fortress Hit by Missile) mine handling (Ship Damage by Mine) and symbol monitoring (Bonus Taken).

Performance on the above measures was obtained from trial 11-12 of each session (when both training conditions received standard game instructions) and was then averaged for each Week. For the two multivariate GLMs, we minimized family-wise type 1 error rate by referencing univariate tests only following a significant multivariate effect. For each multivariate model, we used the approximate $F$ derived from Wilke's $\Lambda$, with $p=0.05$. For each univariate model, we report the uncorrected degrees of freedom although measuring statistical significance at $p=0.05$ after the Huynh-Feldt epsilon correction for violations of the assumptions of the GLM. For the sake of brevity, in our follow-up univariate models, we only report the highest-order interactions that were significant and only reference main effects if no interactions were present. Effect sizes in terms of partial eta squared $\left(\eta_{p}^{2}\right)$ are reported for each effect that reached significance.

\section{TOTAL SCORE}

The first GLM revealed an effect of Week, $F(11,341)=15.58, p<0.001$, $\eta_{p}^{2}=0.34$; Condition, $F(1,31)=4.53, p<0.05, \eta_{p}^{2}=0.13$; and Aiming $F(1,31)=61.51, p<0.001, \eta_{p}^{2}=0.67$. However, there was also an 
interaction between Week and Condition, $F(11,341)=2.71, p<0.05$, $\eta_{\mathrm{p}}^{2}=0.08$ such that participants in the AC and the EC condition started off with similar performance during the first weeks of training $[F(1,32)=1.82, p>0.05]$ but the AC condition (improved more or) did better than the EC condition during the last week of training $\left[F(1,32)=8.06, p<0.01, \eta_{\mathrm{p}}^{2}=0.20\right.$; see Figure 2$]$. There was also an interaction between Week and Aiming, $F(11,341)=8.08, p<0.001$, $\eta_{\mathrm{p}}^{2}=0.21$ such that aiming had more of an effect during the later weeks of training. The three-way interaction between Week, Condition, and Aiming was not significant, $F(1,31)=1.10, p>0.05$.

Taken together, these Total Score analyses suggest that older adults learned to cope with the high demands of the game, improved with practice and that performance changed as a function of the instructions provided during training as well as with subject-specific aiming scores. Moreover, older adults in the AC condition performed better than older adults in the EC condition.

\section{MAIN SUB-MEASURES}

Main sub-measures of SF performance include Points, Control, Velocity, and Speed (see Table 2). The second GLM included these measures into a repeated-measures MANCOVA and revealed a main effect of Week $F(44,1295)=4.44, p<0.001, \eta_{p}^{2}=0.15$ and Aiming $F(4,28)=18.87, p>0.05, \eta_{\mathrm{p}}^{2}=0.73$, but no effect of Condition, $F(4,28)=1.22, p>0.05$. There was a two-way interaction between Week and Condition, $F(44,1295)=1.99, p<0.001, \eta_{\mathrm{p}}^{2}=0.06$ and Week and Aiming $F(44,1295)=4.14, p<0.001, \eta_{p}^{2}=0.12$ as well as a three-way interaction between Week, Condition, and Aiming, $F(44,1295)=1.48, p<0.05, \eta_{\mathrm{p}}^{2}=0.05$.
Follow-up univariate models suggest that the Control and the Speed measure were influenced by subject-specific Aiming scores, $F(1,31)=37.23, p<0.001, \eta_{\mathrm{p}}^{2}=0.55$ and $F(1,31)=31.73, p<0.001$, $\eta_{\mathrm{p}}^{2}=0.51$, respectively. In the Speed measure, there was also an interaction between Week and Aiming, $F(11,341)=2.36, p<0.05$, $\eta_{\mathrm{p}}^{2}=0.06$, such that aiming had more of an effect during the later weeks of training. An interaction between Week and Aiming was also observed in the Points measure, $F(11,341)=7.32$, $p<0.001$, $\eta_{\mathrm{p}}^{2}=0.19$, such that Aiming had more of an effect during the early weeks of training than during the later weeks of training. Note that this is the opposite pattern to the interactions between Week and Aiming that were observed in Speed and Total Score, where aiming had more of an effect during the later weeks of training. In the Velocity measure, there was a three-way interaction between Week, Condition, and Aiming, $F(11,341)=3.62, p<0.05, \eta_{p}^{2}=0.10$. It is difficult to visualize a three-way interaction between two continuous variables. For illustrative purposes, we therefore visualized the nature of this three-way interaction by dichotomizing Aiming in terms of high $(>450)$ and low $(<450)$ aiming scores and graphing velocity for high and low aimers in the EC and AC condition separately (see Figures 3A,B). As is evident from these graphs, velocity in participants with low aiming scores were greater among participants assigned to the AC condition during the early weeks of training, but similar or worse than among participants assigned to the EC condition during the later weeks of training. Velocity in participants with high aiming scores did not differ as a function of training instructions. These main sub-measures analyses suggest that flying the space ship within the hexagon (Control) is influenced

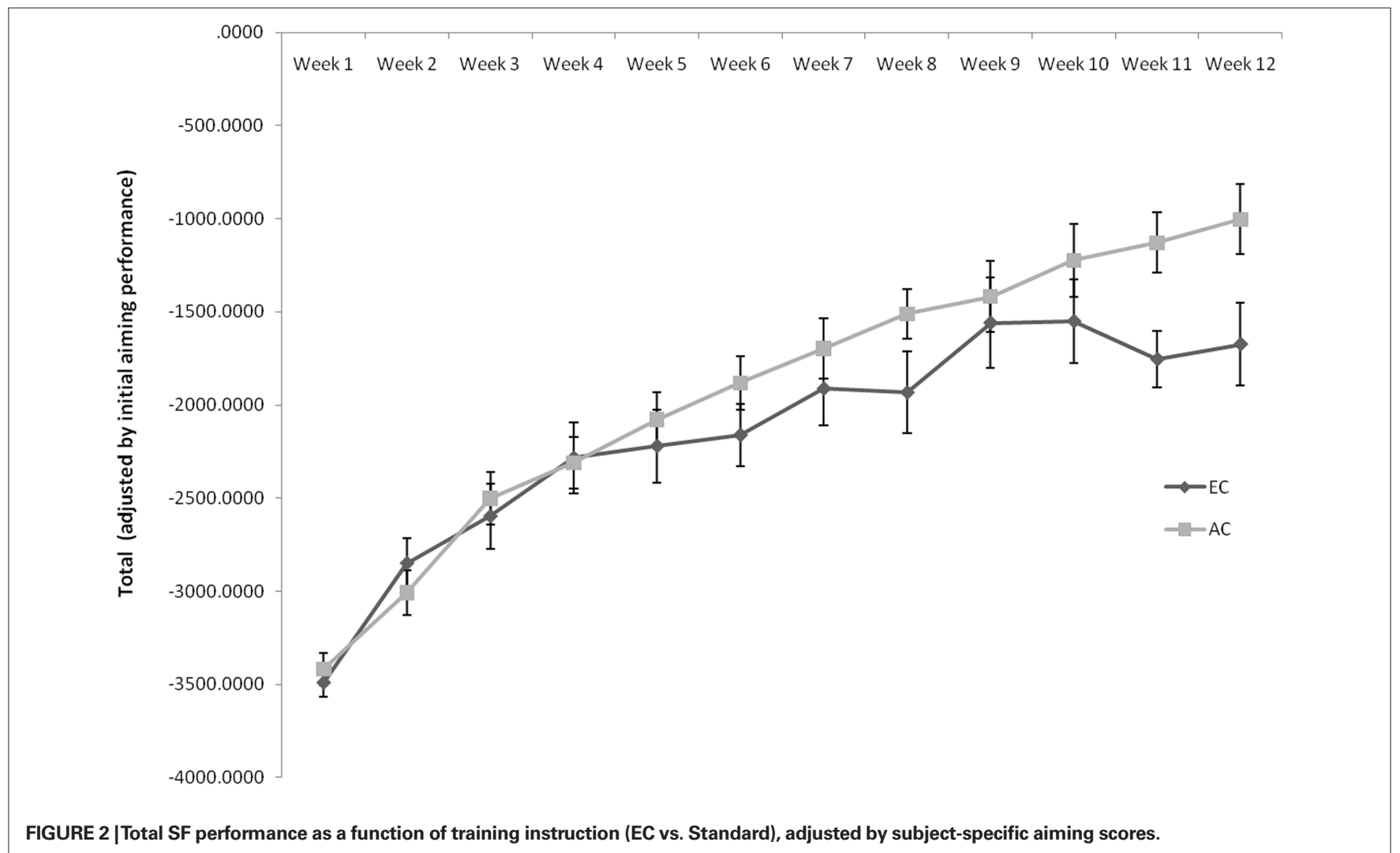


by the subject-specific aiming scores of older adults. The speed or efficiency to detect and destroy mines (Speed) is also influenced by subject-specific aiming scores, particularly during the later weeks of training. The numbers of Points collected from the different task requirements, on the other hand, are influenced by subject-specific aiming scores during the early weeks of training but less so during later the later weeks of training. In order to examine why this is the case, we analyzed each measures that contributes to the Points measure in the upcoming section. Finally, flying the ship slowly within the SF game environment (Velocity), which is related to ship handling and has been shown to be a good learning strategy among younger adults (Frederiksen and White, 1989), improved more in the EC condition for individuals with low subject-specific aiming scores - but not for those with high subject-specific aiming scores. This pattern is consistent with what is observed in younger adults with high basic motor control - whose velocity typically improve more when trained with EC instructions compared to when trained with standard game play instructions (e.g., Gopher et al., 1989). Taken together, these main sub-measure analyses of SF game learning in older adults suggest that training instructions and basic motor control abilities influence the strategies that older adults use when they learn to play the SF game.

\section{POINT-GENERATING SUB-MEASURES}

Point-generating sub-measures include Ship Damage by Fortress, Fortress Destruction, Ship Damage by Mine, Fortress hit by Missile, and Bonus Taken (see Table 2). The third GLM included these measures into a repeated measures MANCOVA and revealed an effect of Week $F(55,1563)=2.37, p<0.001, \eta_{\mathrm{p}}^{2}=0.07$ and Aiming, $F(5,27)=10.10, p<0.001, \eta_{p}^{2}=0.65$ but no main effect of Condition $F(5,27)=1.59, p>0.05$. There was also an interaction between Week and Aiming, $F(55,1563)=6.38, p<0.001, \eta_{p}^{2}=0.17$ and a three-way interaction between Week, Condition, and Aiming $F(55,1563)=1.64, p<0.01, \eta_{\mathrm{p}}^{2}=0.05$. The interactions between Condition and Aiming and between Week and Condition were not significant $(F$ values $<1.14$ ).

Follow-up univariate analyses revealed a three-way interaction between Week, Condition, and Aiming on the ability to score hits against the fortress (Fortress hit by Missile), $F(11,341)=3.92$, $p<0.05, \eta_{p}^{2}=0.11$. We dichotomized Aiming in terms of high and low aiming scores and then graphed Fortress hits by Missiles for high and low aimers in the EC and AC condition separately, to visualize this three-way interaction (see Figures $4 \mathrm{~A}, \mathrm{~B}$ ). As is evident from these graphs, participants with low aiming scores who were assigned to the EC condition and the AC condition displayed similar improvements across training weeks. However, participants with high aiming scores that were assigned to the EC condition, shot more missiles against the fortress than participants with high aiming scores that were assigned to the AC condition, particularly during the later weeks of training. Participants in the AC condition, however, tended to collect more bonus points (Bonus Taken) than participants in the EC condition throughout the 3-month training program, $F(1,31)=4.74, p<0.05, \eta_{p}^{2}=0.013$ (see Figure 5). The ability to destroy the fortress (Fortress Destruction) interacted with Week, Condition, and Aiming, $F(11,341)=4.74, p<0.05, \eta_{\mathrm{p}}^{2}=0.13$. Again, we dichotomized aiming into low and high in order to visualize this three-way interaction (see Figures 6A,B). As is evident from these graphs, Fortress Destruction among high aimers did not differ as a function of Week and Condition. However, among low aimers Fortress Destruction in the EC condition was greater than in the AC condition, particularly during the later weeks of training. No main effects or interactions were observed in the Ship Damage by Fortress measure. In the Ship Damage by Mine measure, however, there was a two-way interaction between Week and Aiming, $F$ (11, $341)=2.71, p<0.05, \eta_{p}^{2}=0.08$ such that aiming had more of an effect during the later weeks of training.

Taken together, these point-generating sub-measure analyses suggest that the learning strategies adopted by older adults during SF game learning are influenced by basic motor ability and by the instructions provided during training. While standard game instructions leads to a strategy that involves collecting bonus points by monitoring dollar signs that randomly appear on the screen, EC instructions leads to a strategy that involves the more difficult aspects of the game such as shooting missiles against the fortress, destroying the fortress, and avoiding damage from mines. Moreover, basic motor control abilities influenced the older adults' ability to shoot missiles against the fortress and to avoid damage from mines, particularly toward the end of the 3-month training program. To confirm that the overall advantage in the AC condition was indeed due to the collection of bonus points, we subtracted the bonus points from the Total Score and re-ran our Total Score Analyses. As expected, we found no difference in Total SF performance between the $\mathrm{AC}$ and the EC condition after this subtraction, $F(1,32)=2.65, p>0.05$.

\section{GENERAL DISCUSSION}

The current study examined if and how cognitively healthy older adults learn to play a complex computer-based action game called the SF, which demands a considerable amount of cognitive control. Taken together, there were three major findings that need to be discussed.

1. Older adults managed to cope, persist, learn, and improve upon their SF game performance.

2. Basic motor ability correlated with SF game performance and learning rate.

3. Standard training instructions lead to better overall SF performance than EC training instructions.

1. Space Fortress game training. Our results clearly show that older adults learn to play the SF game and continue to improve with practice over the course of 3-months although they do not perform at the same level as younger adults. More specifically, while the Total SF score of the older adults in this study was -3453.37 during the first week of training and -1329.25 during the 12 th week of training, a trained young adult control group $(n=7)$ had a Total score of -1613.93 during the first week of training and +1130.93 after the ten sessions (i.e., 2.5 weeks of training). The motivation and the persistence displayed by the older adults in this study is quite remarkable given no, or very limited prior experience with, computer-based action games and, more importantly, the high demands of the SF game. Many older adults in this study never obtained positive performance sco- 
A

Week 1 Week 2 Week 3 Week 4 Week 5 Week 6 Week 7 Week 8 Week 9 Week 10 Week 11 Week 12

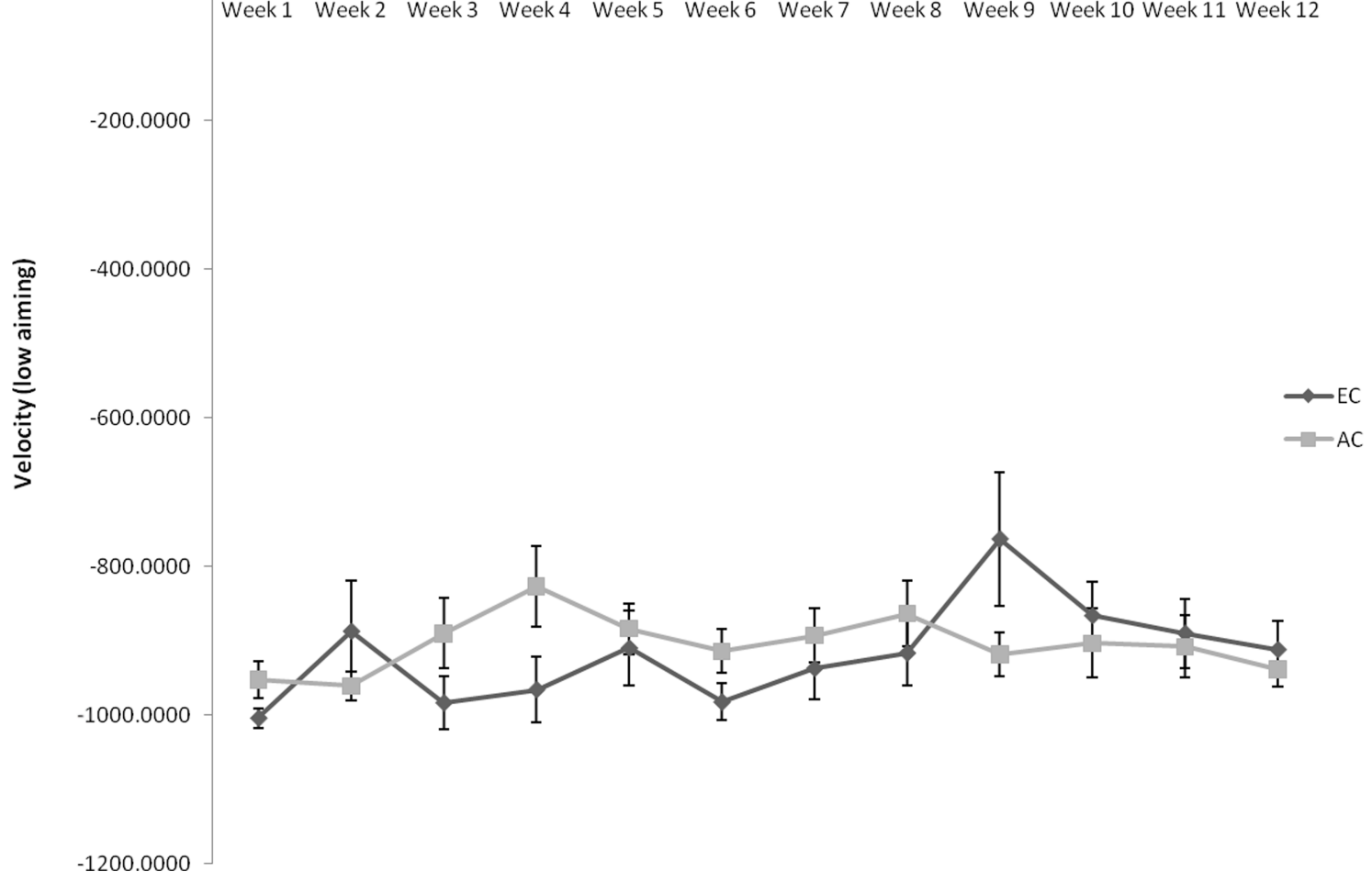

B

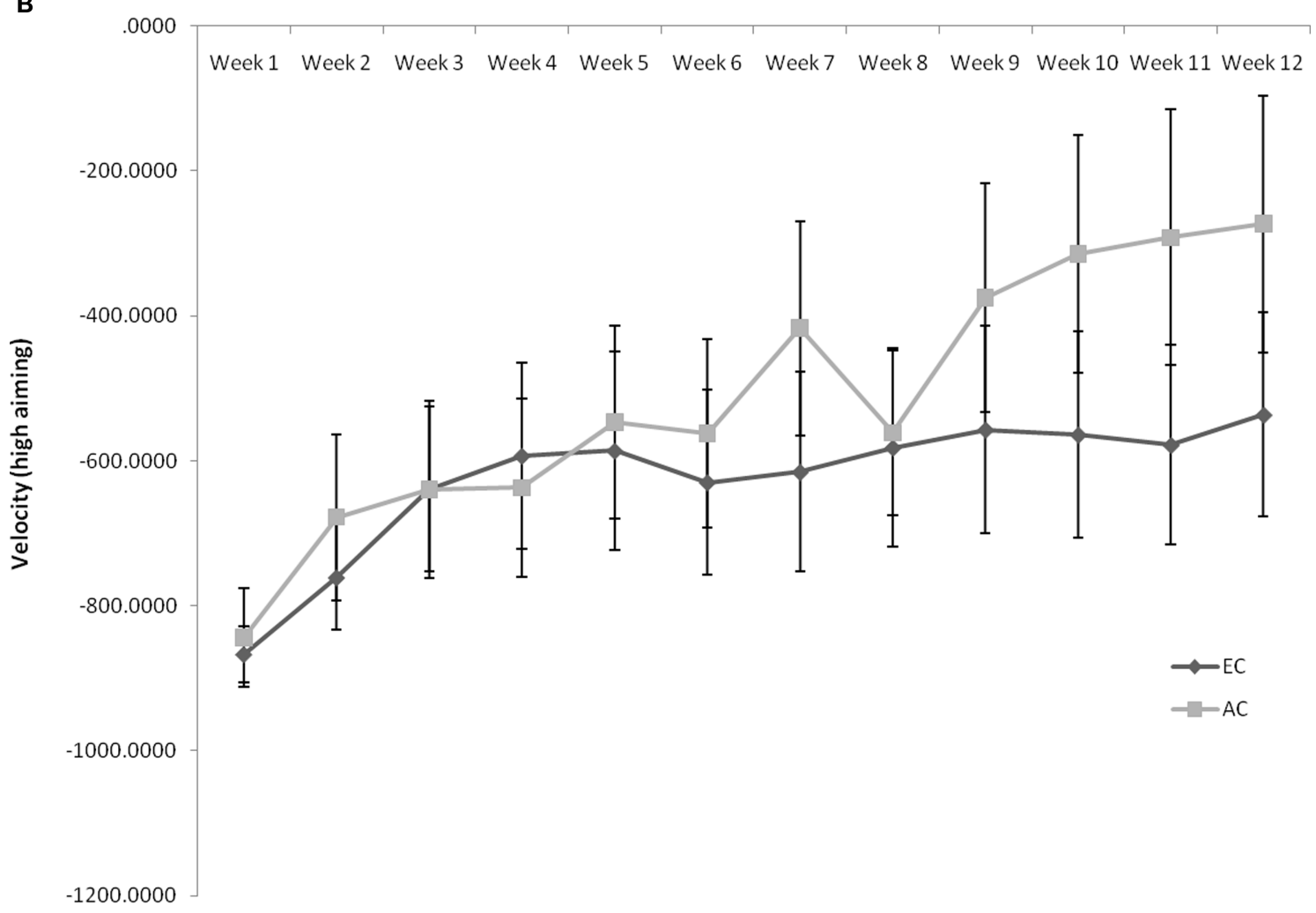

FIGURE 3 | (A) Velocity as a function of training instructions (EC vs. Standard) in participants with low (<450) subject-specific aiming scores. (B) Velocity as a function of training instructions (EC vs. Standard) in participants with high (>450) subject-specific aiming scores. 


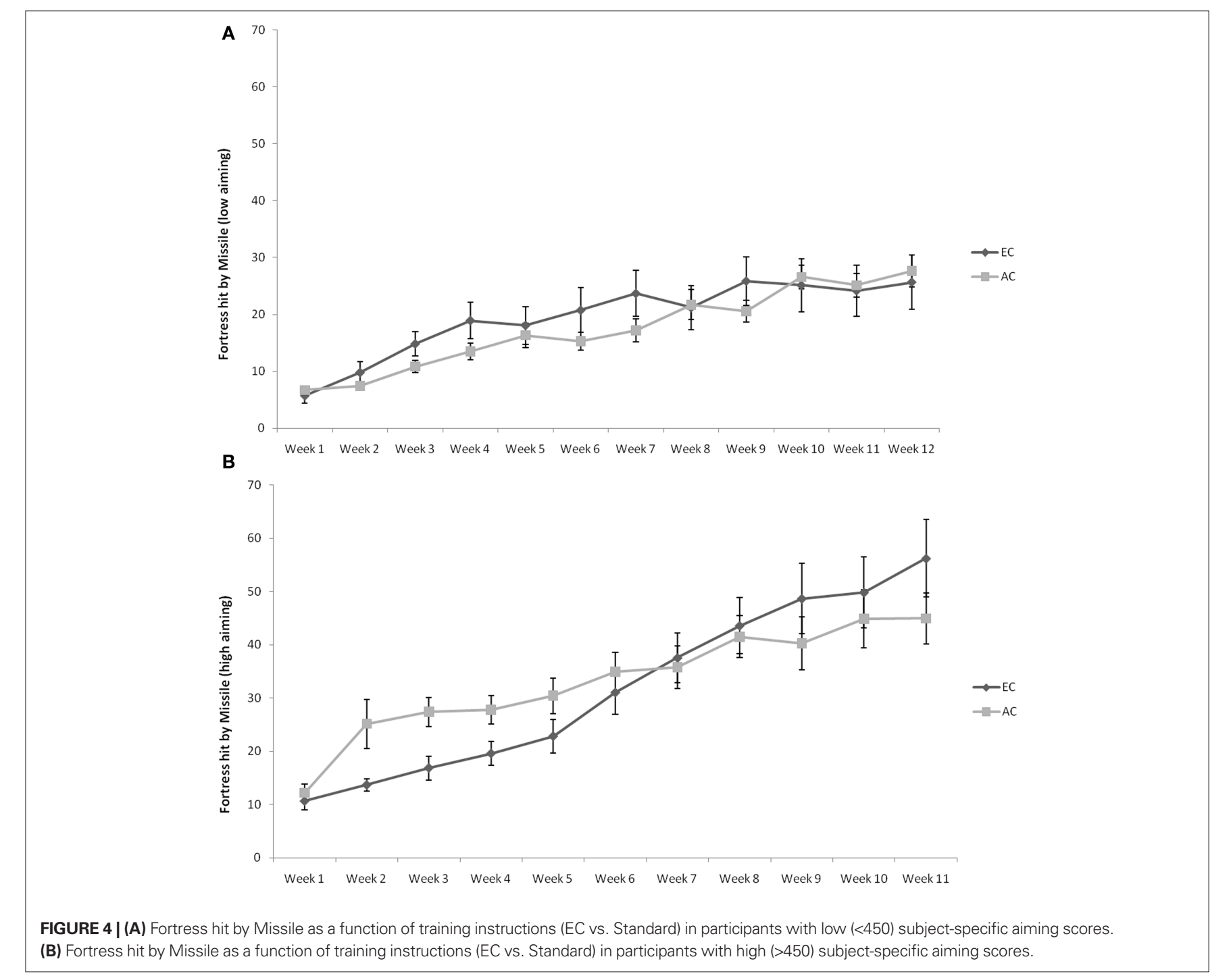

res but most of them completed the full 3 months of SF game training. This is a particularly informative observation when considering the development of this training program as a cognitive intervention that can potentially compensate for age-related cognitive decline. It is also important to note that some older adults managed to obtain positive performance scores toward the end of the training program (e.g., overall SF game performance during Week 12 was positive for two participants in the EC condition and for two participants in the AC condition) and that the overall SF game performance for the highest scoring older adult was 3376.40 during the 12th week of training. While this initial evidence of SF game learning in older adults is promising, future studies will determine if SF game learning promotes transfer of learning to other laboratory tasks and or real-world activities. Given the high cognitive control demands of the SF game, we would predict that SF game learning would be more beneficial to performance on other laboratory tasks or real-world activities that also demand high cognitive control.
2. Basic motor ability. Basic motor ability as measured by the aiming task was shown to be an important determinant of SF game performance and learning rate. The importance of motor control for SF game performance and learning rate is not surprising - as it was already known during the initial series of studies. In fact, this was the original justification for the development of the aiming task and a score of 780 as a threshold entry point for younger adults. In the present study of older adults we administered the aiming task but we did not use this score to select participants or to match their group assignment. In general, aiming task scores of older adults were below the threshold entry point used in studies of younger adults. More specifically, only six older adults in the two groups obtained aiming task scores greater than 780 and the mean aiming task score for the two groups was 515 . By contrast, the mean aiming task score in our young adult control group was 1518. This considerable difference in basic motor ability may have contributed to the lower SF game performance displayed by the older adults in this study. 


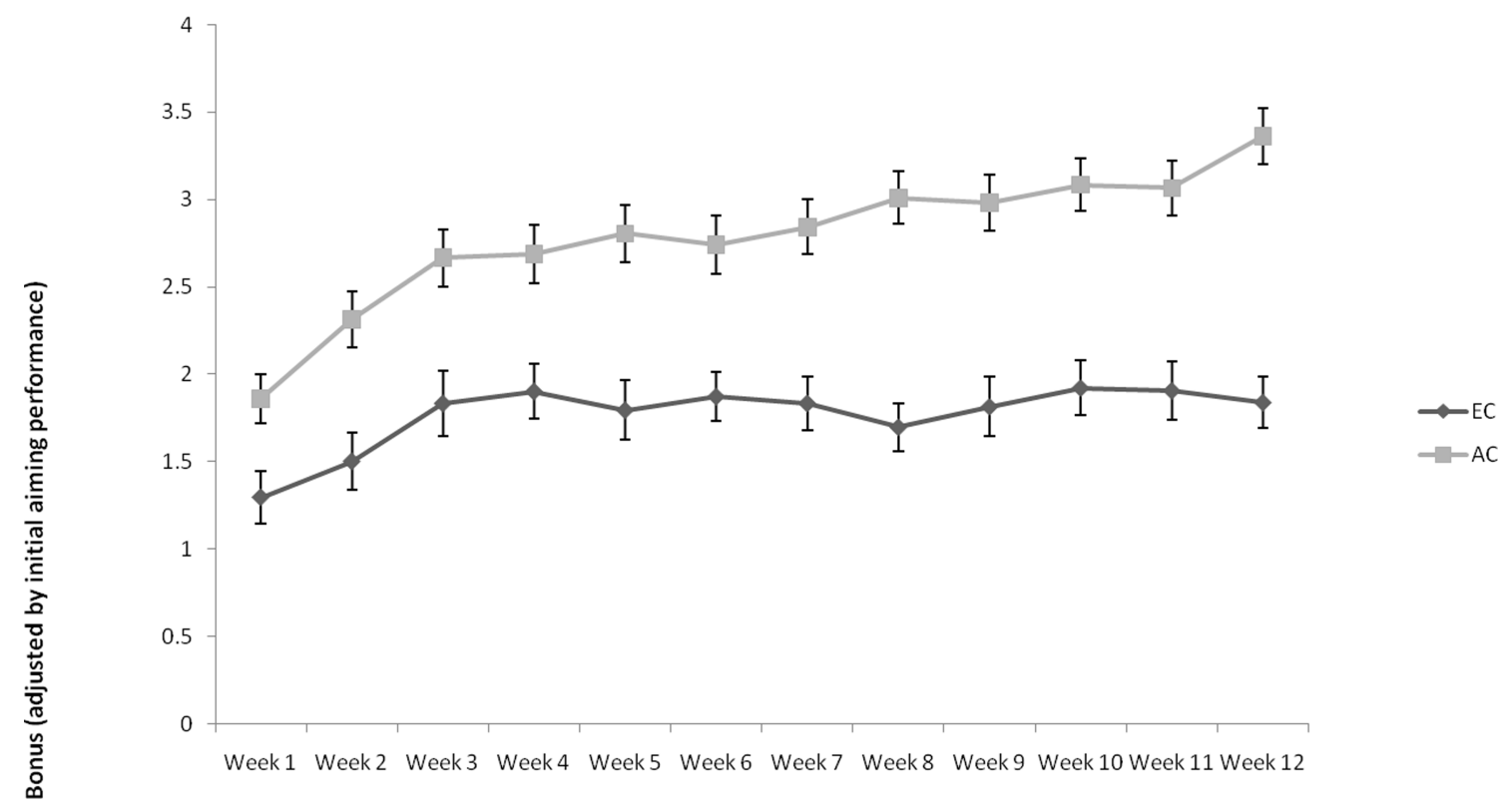

FIGURE 5 | Bonus Taken as a function of training instructions (EC vs. Standard), adjusted by subject-specific aiming scores.

Even though aiming task scores of the older adults in this study were lower than studies of younger adults, post-training analyses showed that they correlated with SF game performance in both training conditions. This finding accentuates the large disadvantage of older adults compared younger adults when learning to play this game. Thus, the fact that overall SF performance is lower in older adults may not only reflect age-related differences in cognitive control and the ability to cope with the high demands of the game, but also large differences in basic motor ability that were due to age and the selection of young adult participants. In this context, it is also important to emphasize that despite this motor disadvantage, older adults were not devastated or completely collapsed. In fact, they coped, learned, improved, and complied with EC instructions. It is hence possible that cognitive control and coping with high demands were practiced and improved, even if SF task performance was low as a consequence of low basic motor ability. This possibility may also shed light onto the interpretation of the contrasting outcome of applying the EC training protocol to older adults.

3. Emphasis Change Instructions. The strong influence of basic motor control ability on SF game learning also speak to the different pattern of results following standard instructions and EC instructions in this study of older adults compared to younger adults (Fabiani et al., 1989; Gopher et al., 1989, 1994; Gopher, 1993, 2007; Erickson et al., 2010). More specifically, while younger adults with high aiming scores were selected in previous SF studies to ensure learning following a small number of SF training sessions, the older adults in this study had lower aiming scores and, therefore, found it difficult to acquire and master the skills that are emphasized by EC instructions - because they demand high motor control. Thus, the additional demands imposed by EC instructions potentially limits SF game learning success. However, our analyses of various sub-measures of SF performance suggest that the overall advantage following standard game instructions compared to EC instructions was primarily due to an increase in the collection of bonus points through symbol monitoring. In other words, older adults trained with standard game instructions learned to monitor dollar signs that randomly appeared on the screen to a much greater extent than older adults trained with EC instructions. However, older adults trained with standard game instructions did not do as well as older adults trained with EC instructions on submeasures related to the primary goal of the game - i.e., shooting and destroying the space fortress. In fact, the number of missiles fired at the fortress, the number of hits at the fortress and the number of fortresses destroyed was greater among older adults that had been trained with EC instructions than among those trained with standard training instructions - as was their ability to avoid damage from mines. It is important to note that the sub-measures that showed greater performance following EC instructions than following standard game instructions are those that involve motor control and are related to ship handling and mine handling, which were emphasized during EC instructions. By contrast, the submeasure that showed greater performance following standard game instruction (Bonus Taken) do not involve motor control and is more related to resource management. Thus, the additional demands imposed by EC instructions may have limited overall SF success but improved performance on some sub-measure related to ship handling and mine handling and, more importantly, precluded the adoption of a simpler strategy such as collecting bonus points. Future studies will determine if SF game learning with EC instructions more effectively promotes transfer of learning to other labo- 


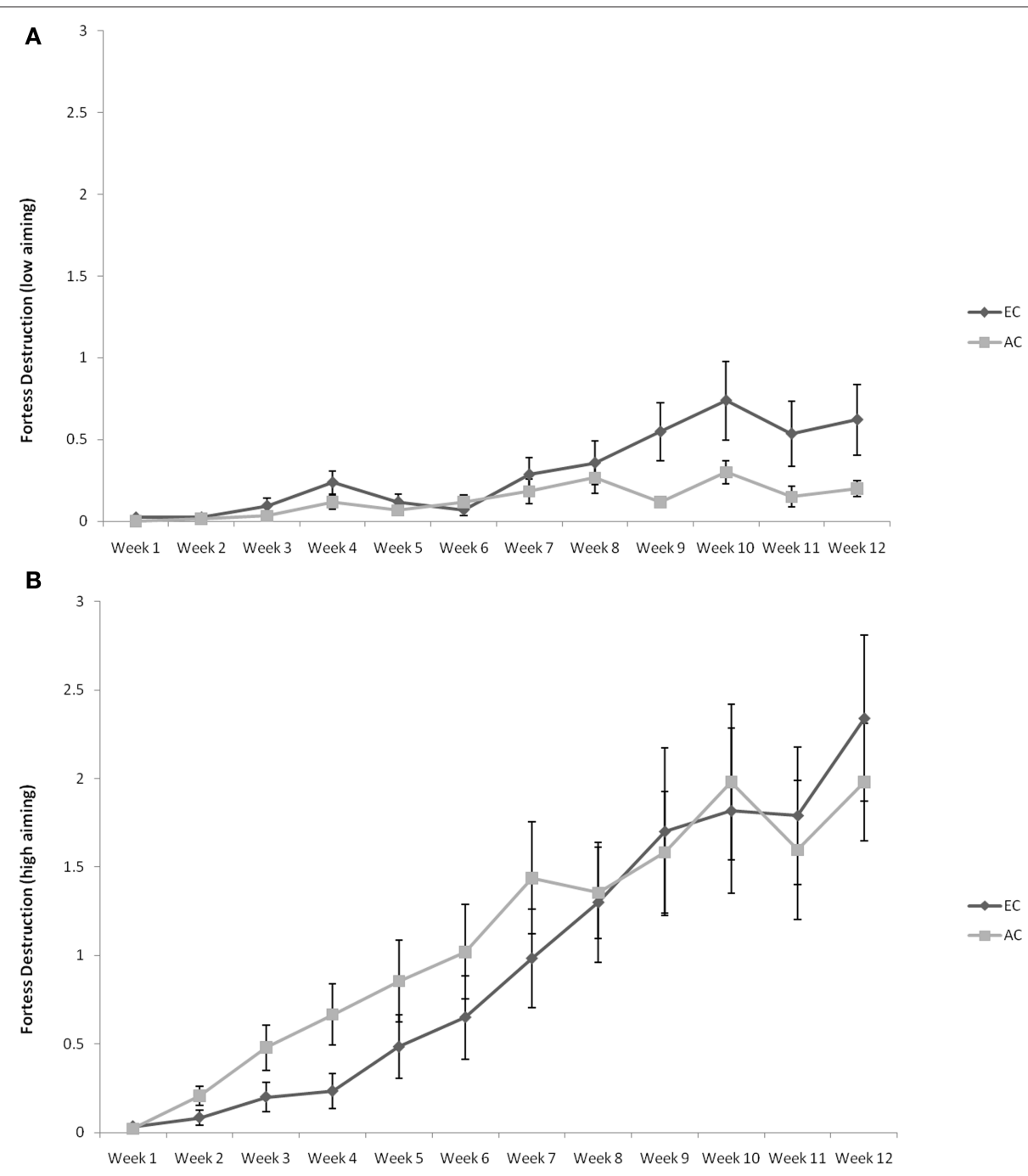

FIGURE 6 (A) Fortress Destruction as a function of training instructions (EC vs. Standard) in participants with low (<450) subject-specific aiming scores. (B) Fortress Destructions as a function of training instructions (EC vs. Standard) in participants with high (>450) subject-specific aiming scores.

ratory tasks and or real-world activities that demand cognitive control. In this context, it is also instructive to point out that studies that have shown an advantage, or equal performance, following EC training instructions and standard training instructions in dual-task performance did not use tasks as complex as the SF game, and did not include such a strong and dynamic control demand (Kramer et al., 1995; Bherer et al., 2005, 2008).

An important result of the applications of an EC training protocol to SF game training in older adults is that participants were able to comply and change their behavior accordingly, even though they were faced with demands that were difficult for them to perform. If EC training focus on the development of cognitive control and participants were able to follow instructions, is it possible that control skills were trained as much or more when basic abilities (i.e., motor control) is low? This question raises an interesting comparison and contrast between training basic abilities and higher-level cognitive control abilities. There are several findings in this study that point to the merit of this direction of study. It is of special significance for maintaining cognitive functions of older adults when basic abilities are degraded.

\section{CONCLUDING COMMENTS}

The current study examined if and how cognitively healthy older adults can learn to play a computer-based action game that place a particularly high demand on cognitive control processes. A comparison between standard and EC instructions were included to examine the effects of further increasing the demand for cognitive control processes during training. The current study is the first to train older adults to play the SF game and findings will be helpful for the further development of this training program as a cognitive intervention that can potentially compensate for age-related cognitive decline. In short, our results suggest that older adults can be trained to play the SF game over the course of 3 months. Moreover, training instructions influenced the game-learning 
strategies that older adults adopt during SF game training and basic motor control influenced how well they learn many different aspects of the SF game. Future studies should study and clarify the confluence between such basic abilities and higher-level of cognitive abilities.

\section{REFERENCES}

Arthur, W. Jr., Strong. M. H., Jordan, J. A., Williamson, J.E., Shebilske, W. L., and Regian, J. W. (1995). Visual attention: individual different in training and predicting complex task performance. Acta Psychol. 88, 3-23.

Basak, C., Boot, W. R., Voss, M. W., and Kramer,A. F. (2008). Can training in a real-time strategy video game attenuate cognitive decline in older adults? Psychol. Aging 23, 765-777.

Belin, D., and Everitt, B. J. (2008). Cocaine seeking habits depend upon dopamine-dependent serial connectivity linking the ventral with the dorsal striatum. Neuron 57, 432-441.

Bherer, L., Kramer, A. F. Peterson, M. S., Colcombe, S., Erickson, K., and Becic, E. (2005). Training effects on dual task performance: are there age-related differences in plasticity of attentional control? Psychol. Aging 20, 695-709.

Bherer, L., Kramer, A. F., Peterson, M. S., Colcombe, S., Erickson, K., and Becic, E. (2008). Transfer effects in task-set cost and dual-task cost after dual-task training in older and younger adults: further evidence for cognitive plasticity in attentional control in late adulthood? Exp. Aging Res. 34, 188-219.

Clark, J. E., Lanphear, A. K., and Riddick, C.C. (1987). The effects of videogame playing on the response selection processing of elderly adults. $J$. Gerontol. 42, 82-85.

Desmond, J. E., \& Fiez, J. A. (1998). Neuroimaging studies of the cerebellum: language, learning and memory. Trends Cogn. Sci. 2, 355-362.

Drew, B., and Waters, J. (1986). Video games: utilization of a novel strategy to improve perceptual motor skills and cognitive functioning in the noninstitutionalized elderly. Cogn. Rehabi. 4, 26-31.

Erickson, K. I., Boot, W. R., Basak, C., Neider, M. B., Prakash, R. S., Voss, M. W., Graybiel, A. M., Simons, D. J., Fabiani, M., Gratton, G., and Kramer, A. F. (2010). Striatal volume predicts level of video game skill acquisition. Cereb. Cortex. 20, 2522-2530.

Fabiani, M., Buckley, J., Gratton, G., Coles, M. G. H., Donchin, M., and Logie, R. (1989). The training of complex task performance. Acta Psychol. 71, 259-299.

Frederiksen, J. R., and White, B. Y. (1989). An approach to training based upon principled task decomposition. Acta Psychol. 71, 89-146.

Goldstein, J., Cajko, L., Oosterbroek, M., Michielsen, M., van Houten, O., and Salverda, F. (1997). Video games and the elderly. Soc. Behav. Pers. 25, 345-352.

Gopher, D. (1993). “The skill of attention control: Acquisition and execution of attention strategies," in Attention and Performance XIV (Cambridge, MA: MIT Press), 299-322.

Gopher, D. (2006). "Control processes in the formation of task units," in Psychological Science Around the World, Vol. 2, Social and Applied Issues, ed. J. Qichen (Hove, UK: Oxford Psychology Press) (A chapter based on a keynote address given at the 28 th International Congress of Psychology), 385-404.

Gopher, D. (2007). "Emphasis change as a training protocol for high demands tasks," in Attention from Theory to Practice, eds A. Kramer, D. Wiegman, and A. Kirlik (New York, NY: Oxford Psychology Press), 209-224.

Gopher, D., Weil, M., and Bareket, T. (1994). Transfer of skill from a computer game trainer to flight. Hum. Factors 36, 387-405.

Gopher, D., Weil, M., and Siegel, D. (1989). Practice under changing priorities: an approach to training of complex skills. Acta Psychol. 71, 147-178.

Green C.S., and Bavelier, D. (2003). Action video game modifies visual selective attention. Nature 423, 534-537.

Green C.S., and Bavelier D. (2006). Effect of action video games on the spatial distribution of visuospatial attention. J. Exp. Psychol. Hum. Percept. Perform. 32, 1465-1478.

Green C. S., and Bavelier, D. (2007). Action-video-game experience alters

\section{ACKNOWLEDGMENTS}

We would like to thank Alexis Richards and Leigh Rich for their research assistance and Brian C. Rakitin for his statistical advice. We would also like to thank the Taub Foundation for providing funding for this research.

the spatial resolution of vision. Psychol. Sci. 18, 88-94.

Green, C. S., and Bavelier, D. (2008). Exercising your brain: a review of human brain plasticity and traininginduced learning. Psychol. Aging 23, 692-701.

Hertzog, C., Kramer, A. F., Wilson, R. S., and Lindenberger, U. (2008). Enrichment effects on adult cognitive development: can the functional capacity of older adults be preserved and enhanced? Psychol. Sci. Public Interest 9, 1-65.

Kramer, A. F., Larish, J. F., and Strayer, D. L. (1995). Training attentional control in dual-task settings: a comparison of young and old adults. J. Exp. Psychol. Appl. 1, 50-76.

Lawrence A. D., Watkins L. H.A., Sahakian B. J., Hodges J. R., and Robbins T. W. (2000). Visual object and visuospatial cognition in Huntington's disease: implications for information processing in corticostriatal circuits. Brain 123, 1349-1364.

Mané,A. M., and Donchin, E. (1989). The Space Fortress game. Acta Psychol. 71, 17-22.

Marie, R. M., Barre, L., Dupuy, B., Viader, F., Defer, G., and Baron, J. C. (1999). Relationships between striatal dopamine denervation and frontal executive tests in Parkinson's disease. Neurosci. Lett. 260, 77-80.

Mattis S. (1976). "Mental status examination for organic mental syndrome in the elderly patient," in Geriatric Psychiatry, eds L. Bellak and T. B. Karasu (New York, NY: Grune \& Stratton), 77-121.

Moscovitch, M., and Winocur, G. (1992). "The neuropsychology of memory and aging," in Handbook of Aging and Cognition, eds F. I. M Craik and T. A. Salthouse (Hillsdale, NJ: Erlbaum Associates), 315-372.

Norman, D. A., and Shallice, T. (1986). "Attention to action: willed and automatic control of behavior," in Consciousness and Self-regulation, Vol. 4, eds R. J. Davidson, G. E. Schwartz, and D. Shapiro (New York, NY: Plenum), 376-390.
Papp, K. V., Walsh, S. J., and Snyder, P. J. (2009). Immediate and delayed effects of cognitive interventions in healthy elderly: a review of current literature and future directions. Alzheimers Dement. 5, 50-60.

Salthouse, T. A., and Somberg, B. L. (1982). Skilled performance: effects of adult age and experience on elementary processes. J. Exp. Psychol. Gen. 111, 176-207.

Shebilske, W. N., Volz, R. A., Gildea, K. M., Workman, J. W., Nanjanath, M. Cao, S., and Whetzel, J. (2005). Revised Space Fortress: a validation study. Behav. Res. Methods 37, 591-601.

Verhaeghen, P., Marcoen, A., and Goossens, L. (1992). Improving memory performance in the aged through mnemonic training: a meta-analytic study. Psychol. Aging 7, 242-251.

Willis, S. L., Tennstedt, S. L., Marsiske, M., Ball, K., Elias, J., Koepke, K. M., Morris, J. N., Rebok, G. W., Unverzagt, F. W., Stoddard,A. M., and Wright, E. (2006). Long-term effects of cognitive training on everyday functional outcomes in older adults. JAMA 296, 2805-2814.

Conflict of Interest Statement: The authors declare that the research was conducted in the absence of any commercial or financial relationships that could be construed as a potential conflict of interest.

Received: 25 March 2010; accepted: 30 September 2010; published online: 11 November 2010.

Citation: Blumen HM, GopherD, Steinerman JR and Stern Y (2010) Training cognitive control in older adults with the Space Fortress game: the role of training instructions and basic motor ability. Front. Ag. Neurosci. 2:145. doi: 10.3389/fnagi.2010.00145

Copyright (c) 2010 Blumen, Gopher, Steinerman and Stern. This is an openaccess article subject to an exclusive license agreement between the authors and the Frontiers Research Foundation, which permits unrestricted use, distribution, and reproduction in any medium, provided the original authors and source are credited. 